\title{
Diffusion in Fluctuating Media: Resonant Activation
}

\author{
Jorge A. Revelli ${ }^{a}$ Carlos. E. Budde ${ }^{b}$ Horacio S. Wio ${ }^{\text {a,c }}$ \\ ${ }^{a}$ Grupo de Física Estadística, Centro Atómico Bariloche (CNEA) and Instituto \\ Balseiro (CNEA and UNC), 8400-San Carlos de Bariloche, Argentina \\ ${ }^{\mathrm{b}}$ Facultad de Matemáticas, Astronomía y Física, Universidad Nacional de \\ Córdoba, 5000 Córdoba, Argentina \\ ${ }^{\mathrm{c}}$ Departament de Física, Universitat de les Illes Balears and IMEDEA \\ (CSIC-UIB), E-07122 Palma de Mallorca, Spain
}

\begin{abstract}
We study the dynamics of a system of particles diffusing in a fluctuating medium: a lattice which can be in two states, with transitions among them induced by a combination of a periodic deterministic and a stochastic process. We characterize the problem studying the mean first passage time (MFPT), the probability of return to the origin $\left(P_{s_{0}}\right)$, and the number of visited lattice sites. We observe a double resonant activation-like phenomenon when we plot the MFPT and $P_{s_{0}}$ as functions of the intensity of the transition rate stochastic component.
\end{abstract}

Key words: Random-Walk, Fluctuating Media, Resonant Activation 46.65.+g, 05.40.FW., 05.10.Ln., 02.50.Eg.

\section{Introduction}

The problem of diffusion in media submitted to global and/or local fluctuations has strongly attracted the attention of researchers. The main motivation was its interest in the study and analysis of a large variety of processes. To name just a few: random-walks in disordered systems [1], ionic conduction in polymeric solid electrolytes [2]; transport of Brownian particles in multi-state media [3]; resonant activation over fluctuating barriers [4] and escape from fluctuating systems [5]; diffusion of ligand with stochastic gating [6]; dynamical trapping problems $[7,8]$, charge transport in molecular scale systems [9], photochemical reactions $[10]$.

Preprint submitted to Elsevier Preprint 16 October 2003 
The above indicated phenomena share the property that the switching between the different configurations or states of the medium is independent of the transport or diffusion processes of the walker. Usually, it is assumed that the states are independent of each other and that the particles are subject to a Markovian process inducing their motions within each state, described by Master or Fokker-Planck equations.

In [11] we have considered the problem of diffusion in fluctuating media subject to Markovian and/or non Markovian switchings, while in [8] we have studied a stochastic resonance-like phenomenon in a gated trapping system. So motivated, we study here the evolution of particles diffusing in a fluctuating medium when the switching mechanism governing the transition between the states has both, a deterministic and a stochastic components. The parameters were chosen in such a way that the effect of the deterministic mechanism alone is not enough to produce the switching. However, the simultaneous action of both signals can induce the jump over the potential barrier and produce the switching in the medium. In this way we expect to capture some basic aspects of the dynamics of more realistic diffusing systems and find situations where the tuning between the stochastic and the deterministic rates can induce some resonant phenomena as in [8].

In order to study this problem we have analyzed, in an infinite system, the Mean First Passage Time (MFPT), the probability that the particle returns to the initial position $\left(P_{s_{0}}\right)$-characterizing in this way the system's capacity to restore its initial condition-, as well as the number of distinct visited lattice sites - a measure of the total length displacement-. The MFPT gives insight into the reaction processes (trapping, etc) and their dependence on the details of the underlying dynamics of the systems, and also provides a useful measure of the efficiency of the trapping, that has been investigated in a broad range of problems from chemistry to biology [12-15].

The main goal of this work is the observation of a double resonant activationlike phenomenon when we analyze the MFPT and the $P_{s_{0}}$ as functions of the noise intensity of the stochastic component of the transition rate among lattice states.

\section{The Model and Monte Carlo Results}

In order to fix ideas we start considering the problem of a particle performing a random walk on a continuous or discrete fluctuating medium characterized by $N$ states which are labelled by the index $j$ (with $j=1,2, \ldots N$ ). Following

van Kampen [16] we define the probability $u_{j}(t)$ that the medium has stayed 
in the $j$ state after a time $t$ since its arrival at $t=0$ as

$$
u_{j}(t)=\exp \left(-\int_{0}^{t} \sum_{i} \gamma_{i j}\left(t^{\prime}\right) d t^{\prime}\right)
$$

where $\gamma_{i j}(t)$ is the probability per unit time for the medium to jump from level $j$ to level $i$ and $t$ is the time it has sojourned in $j$. The switching statistics of the medium $v_{i j}(t)$; defined as the probability that the medium ends its sojourn in the state $j$ after a time between $t$ and $t+d t$ since it arrived at state $j$ at $t=0$ by jumping to a given state $i$; is

$$
v_{i j}(t) d t=u_{j}(t) \gamma_{i j}(t) d t
$$

Equation (2) is completely general and no extra assumption has been made in writing it [16]. Clearly $v_{i j}(t)=v_{j i}(t)$. If the $v_{i j}(t)$ are exponential functions of time, that is the $\gamma_{i j}(t)$ are $t$-independent functions [12], we have a Markovian switching process.

In order to simplify the problem, we assume that the system can fluctuate between only two states. The new aspect here is that we have used a combination of stochastic and deterministic switching mechanisms to describe the transitions between those states. We assume that $v_{i j}(t)$ is given by

$$
v_{i j}(t)=\theta\left[B \sin (\omega t)+\xi-\xi_{c}\right]
$$

where $\theta(x)$ is the step function, and determines when the lattice is in one state or in the other. The dynamics of this mechanism is the following: if the signal, composed of a harmonic part plus the noise contribution $\xi$, reaches a threshold $\xi_{c}$ the lattice changes its state, otherwise it doesn't. We are interested in the case where $\xi_{c}>B$, that is, without noise the deterministic signal is not able to induce a change of the lattice s state. Finally, we assume that $\xi$ is an uncorrelated Gaussian noise of width $\xi_{0}$, i.e. $\langle\xi(t) \xi(u)\rangle=\delta_{t, u} \xi_{0}^{2}$. Note that this is not a standard white noise [13] due to the Kronecker symbol. For each fixed state $j$ of the medium the transport process is assumed Markovian [16].

In [11] we have been able to obtain some analytical results for the MFPT in the case of Markovian and non-Markovian processes. However, and due to the complex dynamics of the present case, we were not able to solve this problem analytically and have resorted to Monte Carlo simulations. The numerical simulations were performed on a one dimensional infinite lattice. The particles were initially located at the lattice site $s_{0}$ in a given state. We assumed that, for each state $j$, the "propagators" $A_{j}[16]$ were arrays $W$ describing a discrete one dimensional random walk in each state of the lattice, and also assumed 
jumps only to first neighbors. The jumps within each state were characterized by two parameters: $\lambda_{j}$, the temporal rate of jump and $\eta_{j}$, the "bias" to make a jump in a given direction. For the return to the origin study $s_{0}=0$. All simulations correspond to averages over $10^{5}$ realizations.

Here we have used state 1 as the "standard" state, characterized by: $\lambda_{1}=1$ and $\eta_{1}=1$ (bias to the left side of the lattice), while state 2 is the test state characterized by $\lambda_{2}=1$. We have chosen the same temporal rates in order to focus on the effect of the bias and the noise intensity on the system response. Hence, $\eta_{2}$ together with $\xi_{0}$, are our control parameters. In addition, and for all cases, we have adopted: $\omega=1, B=1$ and $\xi_{c}=2$.

In Fig. 1 we show the results for the MFPT to reach $s=0$ for a particle that started its motion at site $s_{0}=4$, (a) as a function of the noise intensity $\xi_{0}$, (b) as a function of $\eta_{2}$ and for two values of $\xi_{0}$. From part (a) of this figure it is apparent that there is an optimal value $\xi_{0}$ for which the MFPT has a minimum (the "activation rate" has a maximum). This resonant activation behavior $[4,5]$ is one of the main results of this work. When $\xi_{0}$ is small, the transition rate is low, and the particle is in an "unfavorable" state, remaining there for a long time and contributing to a larger MFPT. When $\xi_{0}$ is large, the transition rate is high, the particle do not shows a net motion, again yielding a large MFPT. But there is an optimal $\xi_{0}$ where the combination between transition rate and motion yields a minimum MFPT. This situation occurs when the bias in each state is strong enough but they are in opposite directions, however the phenomenon disappears when the tendencies in both states are similar. In part (b) we can recognize two main aspects. Firstly, the MFPT decreases as $\eta_{2}$ grows, which is a logical result because increasing $\eta_{2}$ means that we have an increasing bias to the origin. Secondly, there is an important difference in the MFPT for small $\eta_{2}$, whith the noise intensity playing an important role. For small $\xi_{0}$ the transition rate between the states is low and the particle can remain a long time moving to or away from the origin. For large $\eta_{2}$, the differences between the behavior for different noise intensities disappear. Also, at intermediate $\eta_{2}$ values, the MFPT remains higher for a smaller noise intensity.

Figure 2 depicts $P_{s_{0}}$, the probability of return to the origin, (a) as a function of $\eta_{2}$, (b) as a function of the noise intensity $\xi_{0}$ for different values of $\eta_{2}$. The initial condition was $s_{0}=0$, in state 1 , and the simulation time was $10^{4}$. In (a), and for comparison, the case of only one lattice is also included. The latter presents a maximum for an intermediate value of $\eta_{2}$, otherwise the probability decreases as the particle is forced to move away from the origin. For the two state case, we can see that, for small noise intensities, the noise enhances the system response for a fixed (small) value of $\eta_{2}$. This is due to the possibility of changing from the unfavorable state to the one where the particle has a larger probability of moving towards the origin. If we increase the noise intensity, the 

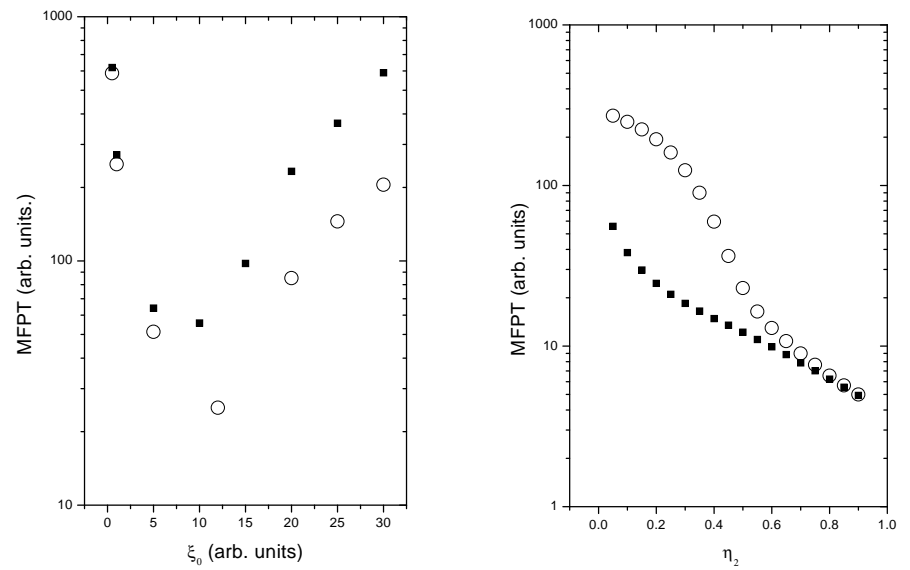

Fig. 1. (a) MFPT vs $\xi_{0}, \bigcirc$ for $\eta_{2}=0.1$ and $\eta_{2}=0.05$. (b) MFPT vs $\eta_{2}$, the bias in state 2. $\bigcirc$ corresponds to the MFPT values for $\xi_{0}=1.0$, and $\boldsymbol{\square}$ for $\xi_{0}=10.0$.
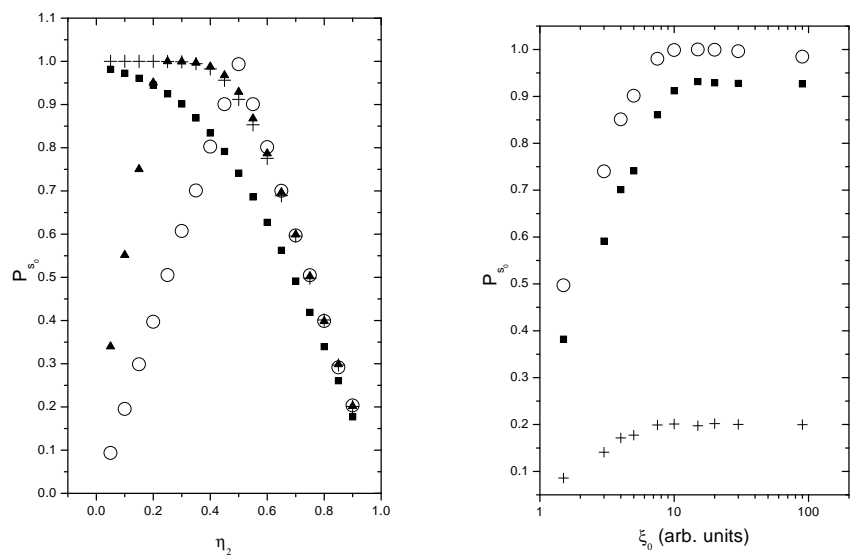

Fig. 2. (a) $P_{s_{0}}$ vs $\eta_{2}, \bigcirc$ represents the one-state case, $\square$ are the data for $\xi_{0}=5.0,+$ for $\xi_{0}=10.0$ and $\boldsymbol{\Delta}$ for $\xi_{0}=20.0$. (b) $P_{s_{0}}$ vs $\xi_{0}, \bigcirc$ represents the data for $\eta_{2}=0.3$, - for $\eta_{2}=0.5$ and + for $\eta_{2}=0.9$

response is enhanced, that is the return probability becomes larger. However, if we continue increasing the noise intensity finally the response for low $\eta_{2}$ is reduced and there appears a maximum in the probability indicating that there is an optimal value of $\eta_{2}$. For large $\eta_{2}$ values, the response becomes smaller for all noise intensities as expected. Here we have another important result of our study, a kind of double resonant behavior both in $\xi_{0}$ and $\eta_{2}$. In (b), the increase of the probability with increasing $\xi_{0}$ is apparent, reaching a kind of plateau for $\xi_{0} \geq 10$. The fact that this probability decreases with increasing $\eta_{2}$ is again a logical result because larger $\eta_{2}$ implies an increasing bias to the origin. 


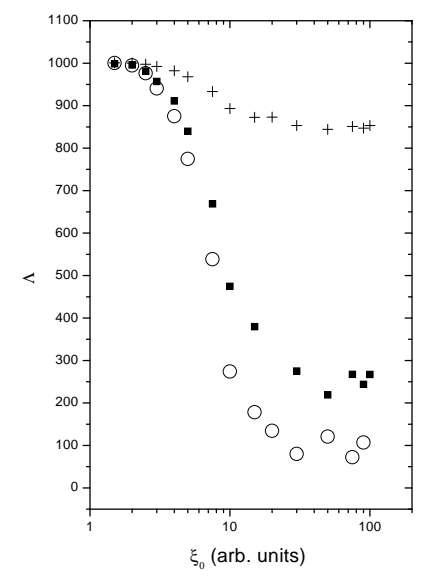

Fig. 3. $\Lambda_{0}$ vs $\xi_{0}$. The simulation time in this and the following figures was $t=1000$. $\bigcirc$ represents the data for $\eta_{2}=0.3, \mathbf{\square}$ for $\eta_{2}=0.5$ and the + for $\eta_{2}=0.9$

Figure 3 shows the total length displacement or number of different lattice sites visited, indicated with $\Lambda$, as a function of the noise intensity $\xi_{0}$, for different values of $\eta_{2}$. For small noise intensity there are no differences among different values of $\eta_{2}$, but for increasing $\xi_{0}, \Lambda$ is strongly reduced for $\eta_{2}=0.3,0.5$, but remains still high for $\eta_{2}=0.9$. This is again consistent with a larger bias to the origin with $\eta_{2}$ large.

Figures 4 (a), (b) and (c), show $\Lambda$ as a function of $\eta_{2}$ and for different values of $\xi_{0}$. In all three cases the simulation time was $t=1000$ (initial state was $1)$. In (a) we compare different cases $\xi_{0}=1.5,10,100$. When $\xi_{0}=1.5, \Lambda$ remains almost constant with $\eta_{2}$. For $\xi_{0}=10$, the curve starts at a small $\Lambda$ and increases monotonically, almost linearly, with $\eta_{2}$. For $\xi_{0}=100$, the presence of a minimum is apparent. A remarkable fact is that before and after the minimum, $\Lambda$ behaves linearly with $\eta_{2}$. It seems that the large $\Lambda$ for small $\xi_{0}$ when $\eta_{2}$ is also small, reduces with increasing $\xi_{0}$ before rising again for still larger values of $\xi_{0}$. This was supported by complementary simulations, see (b), where we have depicted the same situation but now for a lattice with only one state, as well as for the two-state lattice and in the range of large noise intensities $\left(\xi_{0}=20,30,100\right)$. The indicated effect is apparent. For the one-state lattice, the minimum is located at $\eta=0.5$, as it corresponds to the situation of unbiased particle motion. This is a point of symmetry in the sense that a larger or smaller $\eta$ implies a biased motion in one direction or the other (changing $\eta$ by $1-\eta$ only changes the direction of motion but not $\Lambda$ ).

We can see how the noise changes the position of the minimum in the two-state lattice relative to the one-state case. The strong dependence of the system's response for small bias is apparent, as well as the linear scaling of $\Lambda$ with $\eta_{2}$ before and after the minimum. The decrease in the value for small $\eta_{2}$ is also 

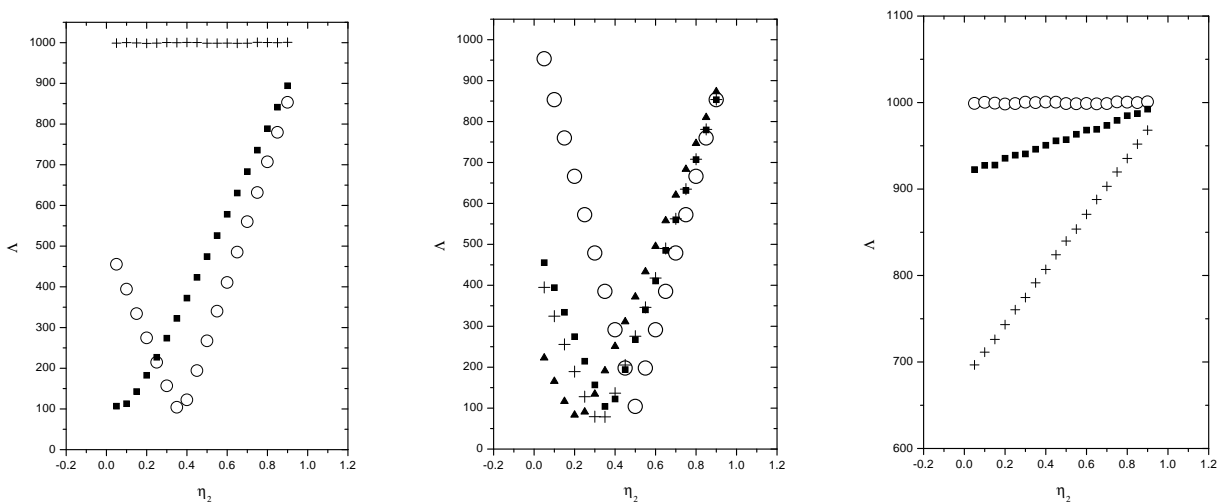

Fig. 4. $\Lambda$ vs $\eta_{2}$. (a) + represents the data for $\xi_{0}=1.5, \boldsymbol{\square}$ for $\xi_{0}=10.0$ and $\bigcirc$ for $\xi_{0}=100.0$. (b) The result for a one-state lattice is indicated by $\bigcirc, \mathbf{\square}$ represents the data for $\xi_{0}=100.0, \boldsymbol{\Delta}$ for $\xi_{0}=30.0$ and the + for $\xi_{0}=20.0$. (c) $\bigcirc$ represents the data for $\xi_{0}=1.5, \boldsymbol{\square}$ for $\xi_{0}=3.0$ and + for $\xi_{0}=5.0$.

shown in part (c) of Fig. 4, where we have depicted the same situation but now for $\xi_{0}=1.5,3$. and 5. . The indicated decrease is clearly seen. We can see that the indicated minimum disappears for small noise intensities, and the "full" linear dependence of $\Lambda$ on $\eta_{2}$ in this range.

\section{Conclusions}

We have studied here the evolution of particles diffusing in a fluctuating medium when the switching mechanism that governs the transition between the states has two components: a deterministic and a stochastic one. We have chosen a periodic signal for the deterministic part while the stochastic component is characterized by a Gaussian white noise of zero mean. The parameters were chosen in such a way that the effect of the deterministic mechanism alone is not enough to produce the switching, but the simultaneous action of the deterministic and the stochastic signals can induce the jump over the potential barrier and produce the switching. The problem was characterized studying the MFPT, analyzing the probability that the particle returns to the initial position, and the number of visited lattice sites.

The main goal of this work is the observation of a resonant activation-like phenomenon when considering the MFPT as a function of the noise intensity of the stochastic component of the transition rate. Another important result is the double resonant behavior, both in $\xi_{0}$ and $\eta_{2}$, observed in Fig. 2. This is another example of the "constructive" role that noise can play, in this case associated to diffusion on a lattice with several (here two) states. 
Acknowledgments: HSW acknowledges the partial support from ANPCyT, Argentine, and thanks the MECyD, Spain, for an award within the Sabbatical Program for Visiting Professors, and to the Universitat de les Illes Balears for the kind hospitality extended to him.

\section{References}

[1] M. Rodriguez, L. Pesquera, M. San Miguel and J. Sancho, J. Stat. Phys. 40, 669 (1985);

A.K. Harrison and R. Zwanzig, Phys. Rev. A 32, 1072 (1985).

[2] A. Nitzan and M.A. Ratner, J. Phys. Chem. 98, 1765 (1994).

[3] H.L. Friedman and A. Ben-Naim, J. Chem. Phys. 48, 120 (1968);

G.H. Weiss, J. Stat. Phys. 8, 221 (1973).

[4] C.R. Doering and J.C. Gadoua, Phys. Rev. Lett. 69, 2318 (1992).

[5] A. Bar-Haim and J. Klafter, Phys. Rev. E, 60, 2554 (1999);

N. Eizenberg and J. Klafter, Physica A 249, 424 (1998).

[6] A. J.A. Mc Cammon and S.H. Northrup, Nature 293, 316 (1981);

T. Novotny and P. Chvosta, Phys. Rev. E 63, 012102 (2000);

A. Szabo, D. Shoup, S.H. Northrup and J.A. Mc Cammon, J. Phys. Chem. 77, 4484 (1982).

[7] J.L. Spouge, A. Szabo and G.H. Weis, Phys. Rev. E 54, 2248 (1996);

M.A. Re, C.E. Budde and M.O. Caceres, Phys. Rev. E 54, 4427 (1996);

Wen-Shyan Sheu; J. Chem. Phys. 110, 5469 (1999).

[8] A.D. Sánchez, J.A. Revelli and H.S. Wio, Physics Letters A 277, 304-309 (2000);

H.S. Wio, J.A. Revelli and A.D. Sánchez, Physica D 168-169C, 167-172 (2002).

[9] J. Jortner and M. Ratner, Molecular Electronics (IUPAC, 1997).

[10] D. L. Jiang and T. Aida, Nature (London) 388, 454 (1997);

D. M. Jounge and D. V. McGrath, Chem. Common. (Cambridge), 857 (1997).

[11] J.A Revelli, C.E. Budde and H.S Wio, Phys. Lett. A 306, 104-109 (2002).

[12] G. H. Weiss, Adv. Chem. Phys 13, 1 (1966); G.H. Weiss, in Aspects and Applications of the Random Walk, (North Holland, Amsterdam, 1994) and references therein.

[13] N.G. Van Kampen, Stochastic Processes in Physics and Chemistry (North Holland, Amsterdam, 1981).

[14] J. Procaccia, S. Mukamel, and J. Ross Chem Phys. 68, 3244 (1978).

[15] V. Seshadri, B. J. West and K. Lindenberg, J. Chem. Phys. 72, 1145 (1980).

[16] N.G. van Kampen, Physica A 96, 435 (1979). 